\title{
The molecular mechanism of mitotic inhibition of TFIIH is mediated by phosphorylation of CDK7
}

\author{
Sasha Akoulitchev and Danny Reinberg ${ }^{1}$ \\ Howard Hughes Medical Institute, Division of N ucleic Acids Enzymology, Department of Biochemistry, Robert Wood \\ Johnson Medical School, University of Medicine and Dentistry of N ew Jersey, Piscataway, N ew Jersey 08854-5635 USA
}

\begin{abstract}
TFIIH is a multisubunit complex, containing ATPase, helicases, and kinase activities. Functionally, TFIIH has been implicated in transcription by RNA polymerase II (RNAPII) and in nucleotide excision repair. A member of the cyclin-dependent kinase family, CDK7, is the kinase subunit of TFIIH. Genetically, CDK7 homologues have been implicated in transcription in Saccharomyces cerevisiae, and in mitotic regulation in Schizosaccharomyces pombe. Here we show that in mitosis the CDK7 subunit of TFIIH and the largest subunit of RNAPII become hyperphosphorylated. MPF-induced phosphorylation of CDK7 results in inhibition of the TFIIH-associated kinase and transcription activities. Negative and positive regulation of TFIIH requires phosphorylation within the T-Ioop of CDK7. Our data establishes TFIIH and its subunit CDK7 as a direct link between the regulation of transcription and the cell cycle.
\end{abstract}

[Key Words: Transcription; TFIIH; CDK7; phosphorylation; cell cycle]

Entry into mitosis is accompanied by repression of transcription by all three nuclear RN A polymerases (Johnson et al. 1965, 1987; Fink et al. 1977). In cultured cells, incorporation of RNA precursors ceases in prophase and resumes at the exit from mitosis. $M$ itotic repression has been associated with a number of regulatory mechanisms (Gottesfeld and Forbes 1997). Accumulating evidence indicates that mitotic repression involves the direct inactivation of key components of the transcription machinery (Gottesfel d et al . 1994). For example, the heptapeptide repeats present in the carboxy-terminal domain (CTD) of the largest subunit of RN A polymerase II (RNAPII) are hyperphosphorylated in meiotic and mitotic cells (Shermoen and O'Farrell 1991; Bellier et al. 1997; Parsons and Spencer 1997). Phosphorylation of the CTD is a critical regulatory step in the modulation of elongation competence (Lu et al. 1991; O'Brien and Lis 1991; Weeks et al. 1993; Dahmus 1996; Zhu et al. 1997). It was found that the CTD is hyperphosphorylated by MPF in vitro resulting in the dissociation of transcription complexes (Cisek and Corden 1989; Zawel et al. 1993). Additionaly, the TAF subunits of TFIID (Segil et al. 1996), and TFIIH are inactivated in mitosis (Long et al. 1998)

A key role in the regulation of the cell cycle is played by a family of cyclin-dependent serine/threonine protein kinases (CDKs) (Reeđ 1992; Coleman and Dunphy 1994; Elledge and Harper 1994; King et al. 1994). These en-

${ }^{1}$ Corresponding author.

E-MAIL reinbed@umdnj.edu; FAX (732) 235-5294. zymes are critical for initiation and completion of DNA replication and cell division in organisms from yeast to mammals (Reed 1992; King et al. 1994). The activity of CDKs is modulated through phosphorylation of their catalytic subunits and by their association with positive (cyclins) and negative regulatory proteins (Elledge and Harper 1994). Distinct cyclins perform different tasks in specific phases of the cell cycle. Entry into mitosis is mediated by $\mathrm{M}$-phase-promoting factor (MPF; Labbe et al. 1989; Gautier et al. 1990). Activation of MPF results in the activation of different kinases and the inhibition of the phosphatases PP1 and PP2A (Kinoshita et al. 1990; $\mathrm{N}$ igg 1993). As a result, multipl e proteins are phosphorylated causing a reorganization of the nuclear envelope, the spindle apparatus, the chromosomes, and the regulation of transcription factors.

We have investigated the mechanisms of repression of transcription by RNAPII during mitosis. Transcription by RN APII requires multiple factors. One family of factors functions to deliver RN A PII to the promoter (TFIIB, TFIID, TFIIF) (Conaway et al. 1990; Lu et al. 1991), whereas two other factors (TFIIE and TFIIH) mediate the escape of RNAPII from the promoter (Goodrich and Tjian 1994; Kumar et al. 1998). These factors are collectively known as the general transcription factors (GTFs) (Roeder 1991; Orphanides et al. 1996). We found that TFIIH and the CTD of RN APII are hyperphosphorylated in mitosis. TFIIH is composed of nine polypeptides with four enzymatic activities (Drapkin and Reinberg 1994; Svejstrup et al. 1996). TFIIH contains a DNA-dependent ATPase activity, two ATP-dependent DNA helicase activities, and a kinase activity specific for the CTD of 
RN APII (Drapkin and Reinberg 1994; Orphanides et al. 1996). TFIIH functions not only in transcription, but al so in nucleotide excision repair (Shaeffer et al. 1993; Drapkin et al. 1994; Sancar 1996). TFIIH exists in two forms, a six subunit core complex that is active in nucleotide excision repair (Svejstrup et al. 1995) and holo-TFIIH that results from the association of core-TFIIH with the kinase complex composed of CDK7, cyclin $\mathrm{H}$, and Mat1 (Drapkin et al. 1996; Reardon et al. 1996). This trimeric complex is known as the cdk-activation kinase (CAK) complex and was initial ly is̄ol ated as an activator of different CDKs (Fisher and Morgan 1994). Holo-TFIIH is necessary for transcription activity (Svejstrup et al. 1995; Drapkin et al. 1996; Reardon et al. 1996; M arinoni et al . 1997; LeRoy et al. 1998), yet the CDK7-kinase activity is dispensable for transcription of some genes in vitro (A Koulitchev et al. 1995; M akela et al. 1995). The regulation of CDK7 (CAK) activity has been the subject of extensive studies (Shuttleworth et al. 1990; Labbe et al. 1994; Tassan et al. 1994; Fisher et al. 1995; Martinez et al. 1997). The activity and substrate specificity of CDK7 is regulated by phosphorylation (Labbe et al. 1994) and/or association with other polypeptides like $\mathrm{M}$ atl and core TFIIH (Devault et al. 1995; Fisher et al. 1995; Adamczewski et al. 1996; Rossignol et al. 1997; Yankulov and Bentley 1997). Genetic studies demonstrate that the S. pombe CDK 7 homolog, crk1/mcs6, is an essential gene with the same substrate specificity as human CDK7. Moreover, $\Delta$ crk 1 cells undergo arrest in late mitosis (Buck et al. 1995). Crk1 was initially identified as the mitotic catastrophe suppressor, mcs6, and could be functionally complemented by Xenopus CDK7, but not by KIN 28 (Cismowski et al. 1995; Valay et al. 1995) the S. cerevisiae homolog of CDK7 (Buck et al. 1995).

Here, we demonstrate that the CTD kinase and transcriptional activities of TFIIH are repressed as the cells enter mitosis. Mitotic repression of TFIIH is mediated through a regulatory phosphorylation of the CDK7 subunit of the CAK complex. This regulation takes place in the T-loop of CDK 7 and it is presumed to induce a conformational change as was described for cdk2 (Russo et al. 1996).

\section{Results}

Mitotic extracts are transcriptionally impaired

To study mitotic repression of transcription and the possible role played by TFIIH, we used HeLa cells arrested with nocodazole. FACS analysis demonstrated that $>95 \%$ of these cells arrested in $\mathrm{G}_{2} / \mathrm{M}$ (Fig. 1b). A mitotic hall mark is the induction of MPF, which is composed of cdc2 and its regulatory subunit cyclin B (N orbury and Nurse 1992). The extracts prepared from mitotic cells displayed $\sim 10$-fold higher $\mathrm{H} 1$ kinase activity than extracts from asynchronous interphase cells (Fig. 1c, lanes 1-2). Affinity purification with immobilized p13 (suc1) protein (Fig. 1c, lanes 3-4; Labbe et al. 1991), followed by Western blot analysis (Fig. 1d), confirmed that almost the entire $\mathrm{H} \mathrm{l}$ kinase activity of the mitotic extract was attributed to an induction of cdc2 activity.

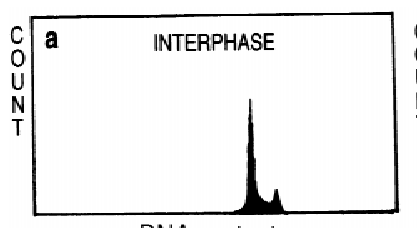

DNA content

C

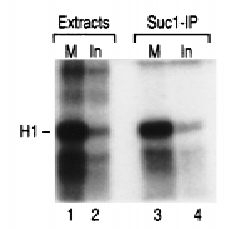

e

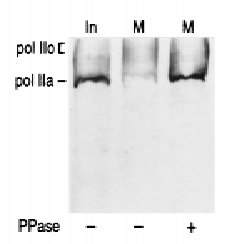

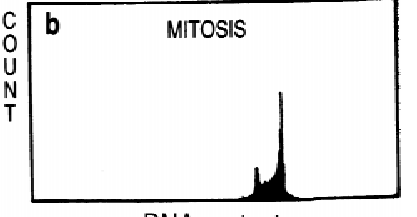

DNA content

d

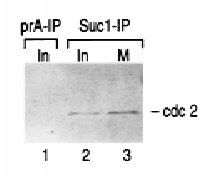

f

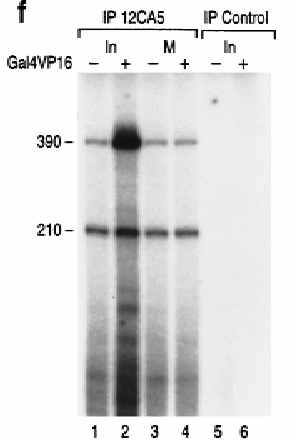

Figure 1. FACS analysis of the asynchronous (interphase) (a) and nocodazole-treated (mitotic) (b) HeLa cells: Distribution of cells in the $G_{1}, S$, and $G_{2} / M$ phases of the cell cycle $(Y$ axis) on the basis of the cellular DN A content (X axis) is plotted on the graph. (c) Histone $\mathrm{Hl}$ kinase assay with extracts derived from the mitotic and interphase HeLa cells (lanes 1,2) or with Suc1(p13)-affinity purified cdc2 kinase from mitotic and interphase extracts (lanes 3,4). (d) Western blot analysis of the Suc1(p13)-affinity purified cdc2 kinase (lanes 2,3) and affinity control (lane 1). (e) Western blot analysis of the CTD of RN APII by use of 8WG16 antibodies (Parsons and Spencer 1997) in the interphase (lane 1) and mitotic extracts (lanes 2,3). (Lane 3) Extracts were pretreated with 10 units of alkal ine phosphatase. (f) Reconstituted basal (210 nucleotides) and activated (390 nucleotides) transcription with eTFIID immunopurified from interphase (lanes 1,2) and mitotic (lanes 3,4) LTR HeLa cells (Zhou et al. 1992). Reactions contained the activator Gal4-VP16 and the coactivators PC4 and TFIIA (lanes 2,4; Ma et al. 1996). The templates used were pG5MLP and pMLP that contain G-less cassettes of different sizes (390 and 210 nucleotides). Transcription was directed by the AdM L promoter. The 390-nucleotide transcript was derived from pG5M LP, which contains five Gal4binding sites upstream of the TATA box. pMLP is devoid of Gal4-binding sites.

Following an earlier report by us performed in vitro (Zawel et al. 1993), and reports of others performed in vivo (Shermoen and O'Farrell 1991; Parsons and Spencer 1997), demonstrating that the cdc2 kinase phosphorylates the CTD of RNAPII (Cisek and Corden 1989), we analyzed the state of phosphorylation of the CTD of RNAPII in interphase and mitotic extracts using monoclonal antibodies that recognize the hypophosphorylated form of RNA PII (IIA). Interphase extracts di splayed a distinct band of $\sim 200 \mathrm{kD}$ corresponding to the hypophos- 
phorylated largest subunit of RN APII, Rpbl (Fig. le, lane 1). This band was not detected in mitotic extracts (Fig. 1e, Iane 2). However, treatment of the mitotic extract with phosphatase restored the hypophosphorylated form (lane 3). It has been shown previously that the hyperphosphorylated form of RN APII is functionally impaired in the formation of the preinitiation complex (Lu et al. 1992; Zawel et al. 1993; Dahmus 1996). Detection of hyperphosphorylation of CTD in mitotic somatic cells in vivo identifies it as one of the potential mechanisms for the mitotic block to transcription (Shermoen and O'Farrell 1991; Parsons and Spencer 1997).

To further characterize the mitotic extract, we anaIyzed the ability of TFIID to function in transcription activation. In agreement with earl ier reports demonstrating that the TAFs are phosphorylated in mitosis resulting in the inhibition of TFIID to function in transcription activation (Segil et al. 1996), we observed that TFIID isolated from nocodazole treated cells by use of an immunopurification procedure (Zhou et al. 1992), was active in a reconstituted assay measuring basal transcription, however, TFIID was impaired in its ability to mediate activation by Gal4-VP16 (Fig. 1f).

The above analyses allow us to conclude that the extracts prepared from nocodazole-arrested cells have all the reported properties of mitotic extracts.

\section{TFIIH activity is impaired in mitosis}

We observed that the transcription activity of the mitotic extract was severely compromised compared with that of the interphase extract (Fig. 2a, cf. Ianes 1 and 2). The transcription observed was mediated by RN APII as it was sensitive to low concentrations $(2 \mu \mathrm{g} / \mathrm{ml})$ of $\alpha$-amanitin (data not shown). N ext, we analyzed whether the purified general transcription factors and/or RNAPII could reactivate the mitotically compromised extract. We observed that the addition of purified TFIIH and RNAPII together effectively restored transcriptional activity (Fig. 2a, lanes 7,8). We found that no other combination of GTFs/RNAPII complemented the mitotic extracts (Fig. 2a, lanes 3-6; data not shown). These finding strongly implicated TFIIH and RNAPII in mitotic inhibition of basal transcription.

$\mathrm{N}$ ext, to directly investigate whether TFIIH was inactivated in the mitotic extracts, we isolated TFIIH from the mitotic extracts and assayed it in a reconstituted transcription system. TFIIH was isolated by an immunoaffinity purification method by use of monoclonal antibodi es against ERCC 3 (LeRoy et al. 1998). A silver staining of a polyacrylamide gel containing a representative TFIIH used in the experiments described bel ow is shown in Figure 2b (see Fig. 2b; M aterials and M ethods). TFIIH isolated from interphase extracts was transcriptionally active (Fig. 2c, Iane 3), whereas TFIIH isolated from the mitotic extract was severely compromised in its ability to reconstitute transcription (Fig. 2c, lane 4). This was not the result of the amounts of purified TFIIH added to the assay as demonstrated by Western blots (Fig 2d).

\section{a}

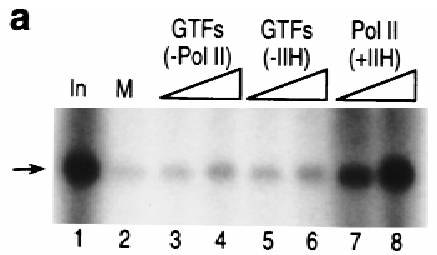

C

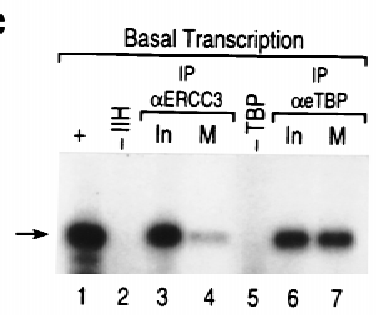

d

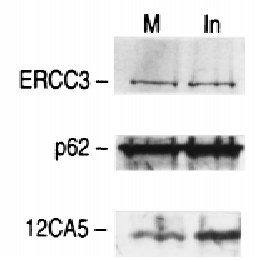

b

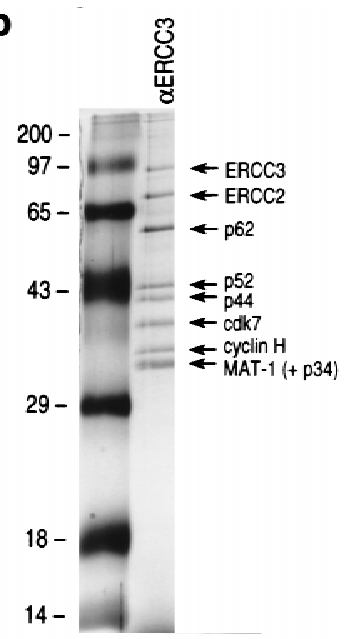

Figure 2. (a) Transcriptional activity of mitotic extracts (lane 2) as compared with the interphase extracts (lane 1) and complemented with different GTFs and RN APII (lanes 3-8). Titration included all GTFs without RNAPII (lanes 3,4 ), GTFs and RNAPII without TFIIH (lanes 5,6), TFIIH and RNAPII (lanes 7,8). (Arrow) The specific transcript derived from the pMLP template. (b) Silver-staining of immunoaffinity purified TFIIH (LeRoy et al. 1998) (lane 2). (Lane 1) M olecular weight markers. (c) Reconstituted basal transcription (lane 1), dependent on TFIIH (lane 2) and TBP (lane 5). Reactions were reconstituted with immunoaffinity purified TFIIH or TFIID from interphase (lanes 3,6 ) or mitotic (lanes 4,7) extracts. (d) Western bl ot of the ERCC 3 and p62 subunits of immunopurified TFIIH and of eT BP (Zhou et al. 1992), used in the reconstituted transcription in C.

M oreover, as an additional control, we isolated TBP from the same extracts using an immunopurification assay (Zhou et al. 1992), and found it to be active in its ability to reconstitute basal transcription (Fig. 2c,d). These re sults collectively demonstrate that TFIIH is impaired in its ability to function in transcription in mitosis. These results are in agreement with previ ous studies performed in vitro that demonstrated that the addition of MPF to interphase extracts resulted in the inactivation of $\mathrm{TFIIH}$ (Long et al. 1998).

\section{The CAK complex is inactivated in mitotic extracts}

Studies performed initially in yeast (Svejstrup et al. 1995), and recently extended to the mammalian factor (Drapkin et al. 1996; Reardon et al. 1996), demonstrate that TFIIH exists in at least two subcomplexes, a complex containing the core subunits of TFIIH (ERCC3, ERCC2, p62, p52, p44, and p34) and devoid of the kinase complex, referred to as core-TFIIH, and a complex associated with the kinase complex (CDK7, cyclin $\mathrm{H}$, and 
a

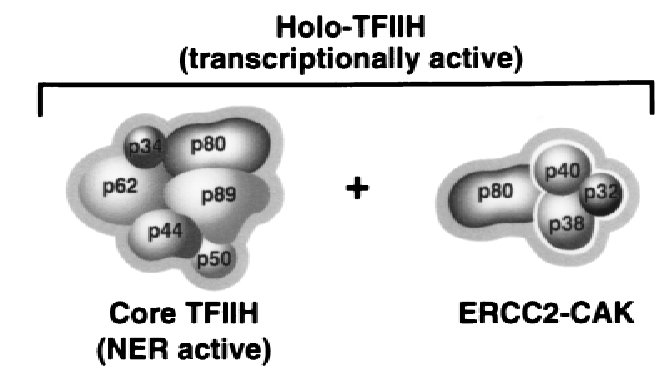

b

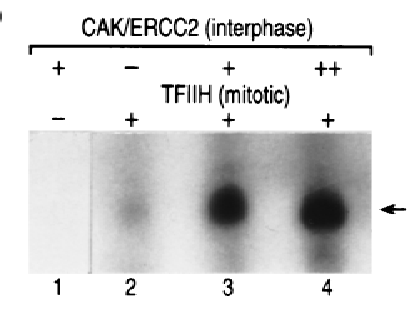

C

TFIIH CAKJERCC2

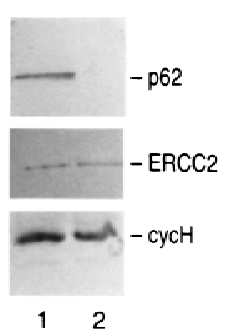

Figure 3. (a) Schematic representation of the composition of core TFIIH, the ERCC2-CAK complex, and holo-TFIIH. (b) Basal transcription by the $A d M L$ promoter was reconstituted with affinity purified mitotic TFIIH (lanes 2-4), CAK/ERCC2 affinity purified from interphase extract (lane 1), or with increasing amounts of the CAK/ERCC2 complex in the presence of mitotic TFIIH (lanes 3,4). (Arrow) The specific transcript derived from the pMLP template. (c) Western blot analysis of the immunoaffinity preparation of mitotic TFIIH and interphase CAK/ERCC2 complexes.

MAT1), referred to as holo-TFIIH (Fig. 3a). Core TFIIH appears to be the form involved in nucleotide excision repair (Svejstrup et al. 1995), whereas holo-TFIIH functions in transcription. Previously, we (Drapkin et al. 1996) and others (Reardon et al. 1996; Marinoni et al. 1997) showed that the CAK complex could be dissociated from holo-TFIIH with high salt. The reassociation of CAK with core-TFIIH restores transcriptional ly active TFIIH (holo-TFIIH) and requires the formation of the intermediary CAK/ERCC 2 complex, which is present in HeLa cell extracts (Drapkin et al. 1996; Reardon et al. 1996; Marinoni et al. 1997). We exploited these features of TFIIH and analyzed whether the transcriptionally compromised mitotic TFIIH could be reactivated by the addition of excess ERCC2-CAK isolated from interphase extracts (Fig. 3c, lane 2). In agreement with the previous results, basal transcription reconstituted with mitotic TFIIH showed reduced transcriptional activity (Fig. 3b, lane 2). On the other hand, when the interphase CAK/ ERCC2 complex was added in lieu of TFIIH, no transcription activity was observed (lane 1), although it was active as a CTD kinase (data not shown). Addition of excess purified interphase CAK/ERCC2 to reactions containing mitotic TFIIH led to restoration of basal transcription (Fig. 3b, lanes 3, 4). These results collectively establish that the CAK-ERCC2 complex is inactivated on entry into mitosis.

The CDK7 subunit is hyperphosphorylated in mitosis

Phosphorylation is an important regulatory modification observed both in mitotic regulation and in regulation of cyclin-dependent kinases. Earlier reports established that CDK 7 is phosphorylated in vivo at two major sites, Ser-164 and Thr-170 (Labbe et al. 1994; Fisher et al. 1995). Phosphorylation on Thr-170 is essential for CDK7 kinase activity in vivo (Labbe et al. 1994), whereas phosphorylation on Ser-164 is di spensi ble and even detrimental in stage VI oocytes (Labbe et al. 1994). The Ser-164 site matches the consensus sequence for cdks/M AP kinases (see Fig. 6a, below). Therefore, we analyzed whether the CDK7 subunit of TFIIH was specifically phosphorylated in mitosis. Interphase and nocodazolearrested HeLa cells were labeled with [32P]orthophosphate in vivo. TFIIH was isolated by immunoprecipitation by use of the ERCC 3 monoclonal antibodies described above (Fig. 2b). The isolated TFIIH complex was then treated with SDS to disrupt the interaction among the different subunits and the CDK7 subunit was immunoprecipitated with antibodies specific to CDK7 (Fig. 4a; $M$ aterials and $M$ ethods). The immunopreci pitates were separated by polyacrylami de gel el ectrophoresis. The au-

a

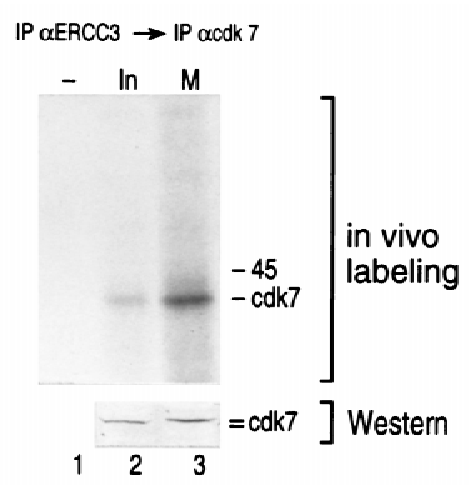

b

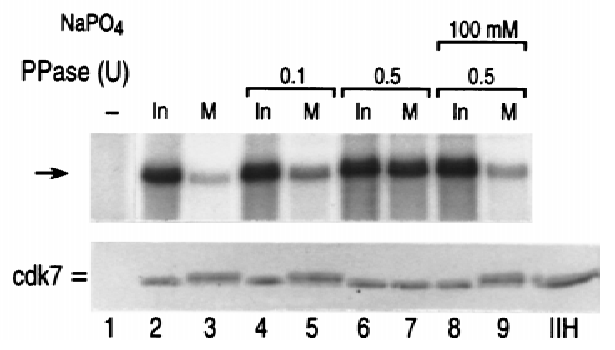

Figure 4. (a) In vivo-labeled CDK7 immunoprecipitated from affinity purified TFIIH and analyzed by Western blot (bottom) after autoradiography (top). ( $\Rightarrow$ The resolved shift in the mobility of mitotic CDK7. (b). (Top) Basal transcription from the AdML promoter was reconstituted with affinity purified interphase TFIIH (lane 2), mitotic TFIIH (lane 3), mitotic or interphase TFIIH pretreated with alkaline phosphatase in the absence or presence of sodium phosphate as indicated. (Lane 1) The result using a mock affinity purification procedure. (Bottom) Western blot analysis of the samples analyzed in transcription at top using anti-CDK7 antibodies. 
CDK7 phosphorylation mediates regulation of TFIIH

toradiograph displayed a single phosphol abel ed polypeptide with increased radioactivity in the mitotic-derived CDK7 sample (Fig. 4a). Western bl ot analysis revealed that the increased radioactivity observed in mitotic-derived CDK7 was not due to differences in the amount of CDK7 loaded on the gels (Fig. 4a, bottom). M ore importantly, a shift in the mobility of the mitotic-derived CDK7 was observed (Fig. 4a, bottom).

The above result prompted us to analyze whether transcriptional activity of mitotic TFIIH could be restored by phosphatase treatment. This approach was possible because in CDK7, the phosphate on Thr-170, which is essential for enzymatic activity (Labbe et al. 1994), is protected from nonspecific dephosphorylation in the CAK complex (Labbe et al . 1994). TFIIH immunopurified from the interphase and mitotic extracts was treated with phosphatase and then used in the reconstituted TFIIHdependent transcription assay (Fig. 4b; see $M$ aterial s and M ethods). We observed that treatment of mitotic TFIIH with phosphatase restored transcriptional activity (Fig. 4b). Importantly, reactivation of TFIIH activity was dependent on the amount of phosphatase added and was blocked by sodium phosphate, a phosphatase inhibitor (Fig. 4b). Western blot analysis of the samples analyzed in transcription demonstrate a shift in the mobility of CDK7 associated with mitotic inactive TFIIH, suggestive that phosphorylation of CDK7 is associated with inactivation of TFIIH activity (Fig. 4b, bottom).

Phosphorylation of CDK7 impairs CTD-kinase and transcription activities

The CTD is one of the substrates of CDK7, and we found that TFIIH isolated from mitotic cell extracts displayed reduced CTD kinase activity (Fig. 5a, Ianes 3,4). The reduced CTD-kinase activity was not due to the amount of factor anal yzed as detected by Western bl ot analysis (Fig. $5 b$, lanes 4,5). We expanded this observation and asked whether inhibition of the TFIIH kinase activity could be reconstituted in vitro. Interphase TFIIH was attached to beads through monoclonal antibodies against ERCC 3 and the beads were then incubated with mitotic- or interphase-derived extracts in the presence of ATP as described in Figure $5 \mathrm{a}$. TFIIH was then recovered from the extracts and analyzed for its ability to phosphorylate the CTD. As shown in Figure 5a, mitotic extract specifically inactivated the TFIIH kinase activity, the interphase-derived extract was without effect (Fig. 5a). The inhibition observed was dependent on ATP (data not shown, see below).

Having established that CDK 7 is hyperphosphorylated in mitosis and that this inhibitory effect can be reconstituted in vitro by incubating interphase TFIIH with mitotic extracts, we attempted to anal yze the si tes phosphorylated in CDK7 by the mitotic extract by incubating bacterially produced recombinant His-tagged CDK7 with interphase and mitotic extracts. Equal amounts (see Fig. 6b) of purified wild-type CDK7 or a mutant form of CDK7, in which the two major phosphorylation sites, Ser-164 and Thr-170, were substituted to alanine were a

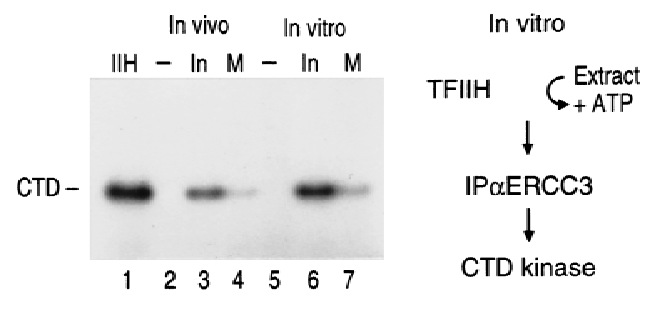

b

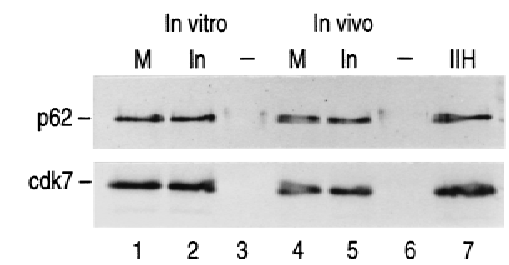

Figure 5. Mitotic TFIIH is deficient in CTD kinase activity. (a) CTD kinase activity of TFIIH directly purified from mitotic and interphase cells (in vivo, lanes 3,4). The kinase activity of conventionally purified TFIIH is shown in lane 1. Purified TFIIH was pretreated with mitotic or interphase extracts in the presence of ATP (in vitro, lanes 6-7, see diagram at right). (b) Western blot analysis of TFIIH subunits (p62 and CDK7) from samples analyzed in a. This blot shows that similar amounts of TFIIH were used in the assay performed in a. The electrophoretic mobility of CDK7 caused by phosphorylation in mitosis is not resolved in this analysis. The samples were separated on a Bio-Rad mini-gel.

incubated in the extracts in the presence of $\left[\gamma-{ }^{32} \mathrm{P}\right] \mathrm{ATP}$. Extensive digestion of the in vitro-labeled CDK7 polypeptides, foll owed by thin-layer chromatography identified two phosphopeptides (Fig. 6c; data not shown). The substitutions in CDK7 abrogated phosphorylation (Fig. 6c; data not shown). This result confirmed earl ier reports that Ser-164 and Thr-170 are the two major sites of phosphorylation with particularly strong phosphorylation of Ser-164 in vivo (Labbe et al. 1994). Comparison of the levels of phosphorylation at these sites revealed $\sim 2$-fold decrease for Thr-170, and 2.5- to 3-fold increase for Ser164 in mitotic extracts.

To analyze more directly whether the phosphorylation of CDK7 is implicated in the regulation of TFIIH, we studied several mutants of CDK7 in vivo. Wild-type CDK7 and individual point mutations with Ser-164 (S) or Thr-170 (T) substituted to alanine were cloned into a mammalian expression vector with an in-frame triple c-M yc tag at the carboxyl terminus (Makela et al. 1997) and were transiently expressed in 293T cells. Following transfection, the cells were treated with nocodazole. cMyc-TAG affinity-purified complexes were further selected for TFIIH by affinity purification using ERCC3 monoclonal antibodies (Fig. 7a). Interphase and mitotic TFIIH were isolated and assayed in transcription and kinase activities (Fig. 7b). In agreement with results presented above, mitotic-derived TFIIH was compromised in its ability to reconstitute transcription and in CTD phosphorylation (Fig. 7b, lanes 1,2). Analysis with mutant TFIIH reveals that both the transcriptional and kinase activities are dependent on phosphorylation of Thr- 
a

cdk 7 DFGLAK'SFGSPNR AYTHQVVTR ${ }^{1704}{ }^{164}$

b

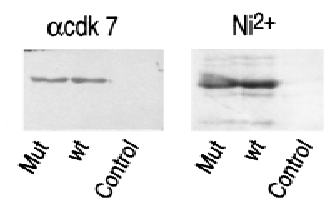

C

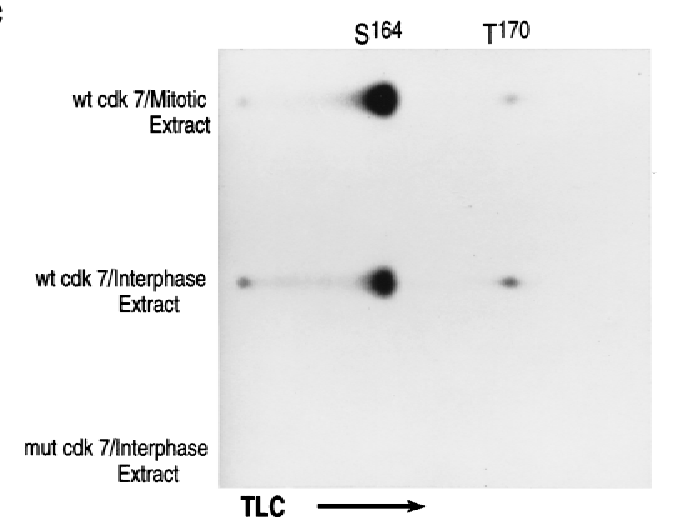

Figure 6. (a) A mino acid sequence of a fragment of CDK7 spanning the sites of phosphorylation in vivo (Ser-164 and Thr-170). (Arrowheads) Positions of cleavage by trypsin. (b) Purified recombinant wild-type and mutant $\left[\mathrm{S}_{164} \mathrm{D}, \mathrm{T}_{170} \mathrm{D}\right] \mathrm{CDK} 7$ containing a carboxy-terminal-His-tag as detected by Western blot using antibodies against CDK7 ( $\alpha-C D K 7)$ and by Coomassie-blue staining $\left(\mathrm{Ni}^{2+}\right)$. (c) Recombinant wild-type and mutant CDK7 were incubated with interphase or mitotic extracts in the presence of $\left[\gamma^{-}{ }^{32}\right]$ ATP. Labeled proteins were affinity purified, digested with trypsin, and resolved by one-dimension thin layer chromatography. Positions of Ser-164 and Thr-170 containing fragments are indicated.

170 (lane 4). Importantly, however, the mutation in Ser164 relieved mitotic repression of TFIIH (lane 3). This experiment demonstrates that phosphorylation on Ser164 is detrimental for TFIIH activity. The studies collectively establish that in mitosis, Ser-164 is phosphorylated and impairs the transcription activity of TFIIH.

\section{Discussion}

Cells arrested in the mitotic stage of the cell cycle display high levels of cdc 2 kinase activity and a high level of protein phosphorylation. Under these conditions, basal transcription by RNAPII is repressed because of the modifications of two factors, RN APII and TFIIH. Detection of the hyperphosphorylated form of RNAPII in mitotic extracts correl ates with earl ier observations in vitro (Cisek and Corden 1989) and studies in Xenopus oocytes (Bellier et al. 1997), Drosophila melanogaster (Shermoen and O'Farrell 1991) and HeLa cells (Parsons and Spencer 1997). It is currently unknown, however, whether inactivation of RN APII activity in mitosis is due to the di rect phosphorylation of the CTD by MPF, as is the case in vitro (Cisek and Corden 1989; Zawel et al. 1993), or whether it involves a downstream cascade of kinases. In metaphase II-arrested Xenopus oocytes, it was shown that inactivation of RN APII results from the activation of the Xp42 MAP kinase (Bellier et al. 1997).

The second factor susceptible to mitotic block is TFIIH. Studies performed with the CDK7 homologs demonstrated its role in negative regulation during meiotic maturation in Xenopus (Shuttl eworth et al. 1990), as well as a mitotic function in Schizosaccharomyces pombe (Buck et al. 1995) and in D. melanogaster (Larochelle et al. 1997). Earlier biochemical analysis of CDK7 regulation did not detect significant changes in its activity during the cell cycle in the context of the CAK complex (T assan et al . 1994; A damczewski et al. 1996). However, previous studies have demonstrated differences in substrate specificity between free CAK and CAK associated with core TFIIH (holoTFIIH) (Rossignol et al. 1997; Yankulov et al. 1997). Our studies uncovered that phosphorylation of CDK7 plays a critical regulatory role within the context of TFIIH. Similar conclusions were reached recently by Long et al. (1998), that demonstrated that the transcription and kinase activities of $\mathrm{TFIIH}$ are negatively regulated in in vitro reconstituted mitotic extracts. Their findings and our conclusions demonstrating that regulation of TFIIH dependent on the association of the CAK complex with core TFIIH are in agreement, yet in the studies of Long et al . (1998), the molecular mechanism of inhibition, through CDK7, was not analyzed.

Extensive studies of CDK7 regulation reveal pathways either common for other members of the CDK family or unique for CDK7. For example, as is the case with other CDKs, the kinase activity of CDK 7 requires its association with a cyclin partner, cyclin H (Labbe et al. 1994; Martinez et al. 1997). Moreover, full activity in vivo requires phosphorylation of a specific residue within the T-loop (Thr-170; Labbe et al . 1994). On the other hand, in vitro, the association of the Matl subunit of CAK with the CDK7/cyclin $\mathrm{H}$ complex can confer activity to CDK7, not requiring prior phosphorylation (Fisher et al. 1995; Martinez et al. 1997). Ser-164 within the T-loop of CDK 7 corresponds to the cdk/M AP kinase phosphorylation consensus site and is responsible for specific phosphorylation of CDK7 in mitosis and for negative regulation of TFIIH activity. The presence of a cdk/MAP kinase consensus sequence within the $T$-loop is unique to CDK7. As a phenomenon, inhibitory phosphorylation within the T-loop of a kinase has been described (Luo and Lodish 1997). Collectively, these results demonstrate that the transcriptional and kinase activities of TFIIH depend on the phosphorylation state of its CDK7 subunit in vivo.

Previous studies demonstrated that the CDK7-kinase activity is dispensable for transcription of one class of promoters (represented by the TATA-containing AdMLP) (Akoulitchev et al . 1995; M akela et al. 1995), but is required for transcription from other classes of promoters (represented by the TATA-less DHFR promoter) (Akoulitchev et al. 1995). Interestingly, in the case of the DHFR promoter, it was found that the requirement for 
a

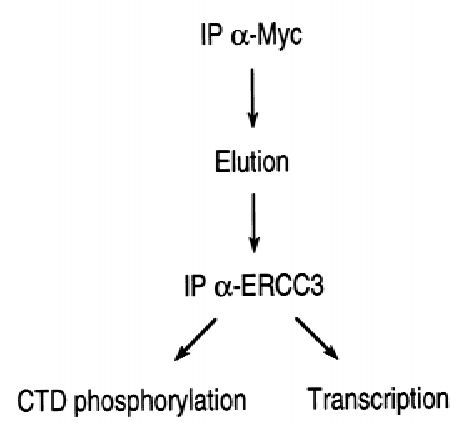

b

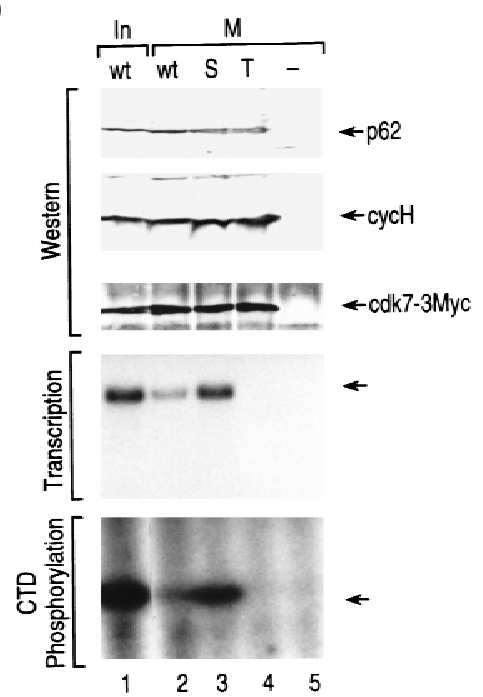

CDK7 phosphorylation mediates regulation of TFIIH

Figure 7. (a) Schematic representation of the procedure used to isolate TFIIH from transfected cells containing mutated subunits of CDK7. TFIIH containing c-Myc-tagged CDK7 was isolated from interphase and mitotic cells. After cell lysis, the transfected CDK7 subunit was recovered by immunoprecipitation by use of antibodies recognizing the c-myc tag. The IPs were eluted and the transfected myc-tagged CDK7 subunits were sel ected for those subunits that were incorporated into TFIIH by performing a second immunoprecipitation with anti-ERCC3 monoclonal antibodies. The IPs were functionally analyzed in transcription and CTD phosphorylation. (b) TFIIH isolated from mitotic cells and containing a mutation in Ser-164 [Ser ${ }_{164} A(S)$ ] or a mutation in Thr-170 $\left[T h r_{170} A(T)\right]$ or a control vector were assayed for transcription and kinase activities. The amount of TFIIH recovered and used in the functional analysis was analyzed by Western blot by use of antibodies against the p62, cyclin $\mathrm{H}$, and CDK7-[3xMyc] subunits (top). The samples were separated on a Bio-Rad minigel; therefore, the change in electrophoretic mobility due to phosphorylation of CDK7-[3xMyc] could not be resolved. Functionally, the mutant proteins were assayed in basal transcription (middle), and for CTD-kinase (bottom) activities. M inus ( $\rightarrow$ abovethe lane denotes the activity present in cells that were transfected with the M yc tag but devoid of the CDK7 coding sequences (empty vector).

the CTD (the substrate for the kinase) was during complex assembly and/or first bond formation, and preceded the requirement for the CDK7 kinase activity that was found to be at a later step. Regardl ess of the requirement for the CDK7-kinase activity, the CDK7 polypeptide, together with cyclin $\mathrm{H}$ and $\mathrm{Mat}$, are necessary for the transcriptional activity of TFIIH (Drapkin et al. 1996; Reardon et al. 1996; Marinoni et al. 1997). Although the inhibition of the CDK7-kinase activity, by mutations in the ATP-binding domain in the catalytic cleft, is not sufficient to compromise the transcriptional activity of TFIIH on the AdML promoter (Akoulitchev et al. 1995; Makela et al. 1995), we would like to suggest that negative regulation of the TFIIH transcription activity in mitosis is mediated via a conformational change of CDK7. The changes are mediated by phosphorylation of residues within the T-loop (Russo et al. 1997). It remains unclear as to which of the kinase(s) acts directly upstream from CDK7. Also, the relationship between the Thr-170 and Ser-164 phosphorylation pathways needs to be elucidated.

In conclusion, our studies demonstrate that mitotic repression of basal transcription results from the phosphorylation of RNAPII and TFIIH. Others have demonstrated previously that phosphorylation of the TAF subunits of TFIID in mitosis impairs activated transcription (Segil et al. 1996). These studies collectively demonstrate that the cell has developed mechanisms to silence transcription during mitosis, by affecting different steps of the transcryption cycle, that is, initiation via the TAF subunits of TFIID and CTD phosphorylation, promoter escape via the CDK7 subunit of TFIIH, and perhaps elongation, by extensive phosphorylation of the CTD by MPF or MPF-activated kinase.

\section{Materials and methods}

Cell culture and in vivo labeling

Long terminal repeat HeLa cells containing epitope-tagged TATA-binding protein (TBP; Zhou et al. 1992) were grown in suspension in 10\% DMEM. Mitotic arrest was obtained by applying $400 \mathrm{ng} / \mathrm{ml}$ nocodazole to 30 liters of growing cell culture $\left(2 \times 10^{10}\right.$ cells) $24 \mathrm{hr}$ prior harvesting. [32 P]Orthophosphate (1 $\mathrm{mCi}$ ) was applied to actively growing cells $16 \mathrm{hr}$ before harvesting.

\section{Extract preparation}

Whole-cell extract was prepared as described (Manley et al. 1980) from $2 \times 10^{10}$ cells with addition of $20 \mathrm{~mm}$ EGTA, $80 \mathrm{~mm}$ $\beta$-glycerophosphate ( $\mathrm{pH} 7.3$ ), $10 \mathrm{~mm}$ sodium fluoride, $10 \mathrm{~nm}$ ocadaic acid, $100 \mathrm{~nm}$ calyculin A, and $1 \mathrm{~mm}$ ATP. Ocadaic acid and calyculin A were maintained in the extracts through purification.

Immunoprecipitation, immunoaffinity purification, and functional assays

Immunoaffinity purification of TFIIH with ERCC 3 monoclonal antibodies was performed as described (LeRoy et al. 1998). In short, proteins from the second step of purification of TFIIH, the DEAE-cellulose bound fraction, were incubated with protein A-immobilized anti-ERCC 3 monoclonal antibodies. The beads were washed extensively with $\mathrm{BC} 400(400 \mathrm{~mm} \mathrm{KCl}, 10 \mathrm{~mm}$ Tris$\mathrm{HCl}$ at $\mathrm{pH} 7.9,0.1 \mathrm{~mm}$ EDTA, 20\% glycerol) and TFIIH was eluted with a peptide. Immunoaffinity purification of CAK/ ERCC2 was as follows: The same protein fraction used above was incubated with protein A-immobilized cyclin $\mathrm{H}$ monoclonal antibodies. The beads were washed extensively in BC900 (900 mM KCl; the rest as in BC 400) to dissociate core TFIIH. The CAK complex was eluted with $1.0 \mathrm{~m}$ ammonium sulfate, $50 \%$ ethylene glycol, $10 \mathrm{~mm}$ Tris- $\mathrm{HCl}$ (pH 7.9). Double-immunoaf- 
finity purification of in vivo labeled CDK7 was performed as follows: After immunoprecipitation of TFIIH with anti-ERCC3 antibodies the samples were heated at $100^{\circ} \mathrm{C}$ for $5 \mathrm{~min}$ in BC 100, 1\% SDS, diluted 10-fold in RIPA buffer $(0.15 \mathrm{M} \mathrm{N} \mathrm{aCl}, 50$ $\mathrm{mm}$ Tris- $\mathrm{HCl}$ at $\mathrm{pH} 7.5,0.5 \% \mathrm{NP}-40,0.1 \%$ SDS) and used in immunoprecipitation with anti-CDK7 antibodies immobilized on protein $\mathrm{A}$ beads. No other subunits of TFIIH coimmunoprecipitated with CDK7 in this procedure. Immunoprecipitates of TFIIH and epitope-tagged TBP (eTBP, eTFIID) used in transcription/kinase reactions were carried out as follows: $50 \mu \mathrm{l}$ of protein $\mathrm{A}$ beads were saturated with the corresponding antibodies for $2 \mathrm{hr}$ at $4^{\circ} \mathrm{C}$, washed extensively with PBS, and incubated with $500 \mu \mathrm{l}$ of the extracts for $2 \mathrm{hr}$ at $4^{\circ} \mathrm{C}$; washed four times with $20 \mathrm{~mm}$ Tris- $\mathrm{HCl}$ (pH 7.9), $150 \mathrm{~mm} \mathrm{~N} \mathrm{aCl}, 2$ mm EDTA, 10 $\mathrm{mM} \beta$-glycerol phosphate, $5 \mathrm{~mm}$ sodium fluoride, $10 \mathrm{~nm}$ ocadaic acid, and $100 \mathrm{~nm}$ calyculin A; and washed two times in transcription or kinase buffer containing $10 \mathrm{~nm}$ ocadaic acid. Protein A beads $(10 \mu \mathrm{l})$ were added to transcription/kinase reactions. Reconstituted transcription reactions with the AdMLP were performed as described (A koulitchev et al . 1995). Reactions performed with the extracts were as described, but the interphase and mitotic extracts were pretreated with 10 units of hexokinase (Boehringer) to remove residual ATP. Reactions were performed under single-round transcription conditions by using a pulse-chase protocol: preinitiation complexes were formed for 1 $\mathrm{hr}$, followed by the addition of ATP and UTP (500 mM), and $\left[\alpha^{-}{ }^{32} \mathrm{P}\right]$ CTP $(1 \mu \mathrm{M})$ for $5 \mathrm{~min}$; followed by the addition of $500 \mathrm{~mm}$ CTP for a 15 min chase.

\section{Phosphatase treatment}

Immunopurified TFIIH from $500 \mu$ of interphase or mitotic extracts was incubated with $0.1-0.5$ units of alkaline phosphatase for $15 \mathrm{~min}$ at $25^{\circ} \mathrm{C}$ in the absence or the presence of $100 \mathrm{~mm}$ sodium phosphate. Phosphatase treatment was performed on TFIIH attached to the beads. This allowed the removal of the phosphatase and/or phosphate prior to the addition of TFIIH to the assays.

\section{Western blot}

Western blots with antibodies against the CTD of RNAPII (8WG 16), the HA tag of eTBP (12CA5), ERCC3, ERCC2, p62, cyclin $\mathrm{H}$, or CDK7 were carried out with PVDF membranes (Bio-Rad) following the manufacturer's recommendations.

\section{Kinase assay}

Kinase reactions were performed in a $40 \mu \mathrm{l}$ volume containing $80 \mathrm{~mm}$-glycerolphosphate (pH 7.5), $20 \mathrm{~mm}$ EGTA, $15 \mathrm{~mm}$ $\mathrm{M} \mathrm{gCl}_{2}, 1 \mathrm{~mm}$ DTT, $1 \mathrm{~mm}$ ATP, $10 \mu \mathrm{Ci}\left[\gamma^{-32} \mathrm{P}\right] \mathrm{ATP}$, and $1 \mathrm{mg} / \mathrm{ml}$ of CTD peptide or histone $\mathrm{H} 1$. Reactions were incubated for 15 min at $30^{\circ} \mathrm{C}$. An aliquot $(5 \mu \mathrm{l})$ was analyzed by electrophoresis on $15 \%$ polyacrylamide-SDS gels.

\section{Acknowledgments}

We thank Gary LeRoy for assistance in the affinity purification of TFIIH. Weal so thank the members of the Reinberg laboratory for hel pful suggestions and to Drs. Mike Hampsey, Jim M anley, Ron Morris, and George Orphanides for comments on the manuscript. This work was supported by grants from the $\mathrm{Na}$ - tional Institute of Health (GM-37120 and GM-48518) and from the Howard Hughes Medical Institute to D.R.

The publication costs of this article were defrayed in part by payment of page charges. This article must therefore be hereby marked 'advertisement' in accordance with 18 USC section 1734 solely to indicate this fact.

\section{References}

Adamczewski, J.P., M. Rossignol, J.-P. Tassan, E.A. Nigg, V. Moncollin, and J.M. Egly. 1996. MAT1, cdk7 and cyclin H form a kinase complex which is UV light-sensitive upon association with TFIIH. EMBO J. 15: 1877-1844.

Akoulitchev, S., T.P. M äkelä, R.A. Weinberg, and D. Reinberg. 1995. Requirement for TFIIH kinase activity in transcription by RN A polymerase II. Nature 377: 557-560.

Bellier, S., M.F. Dubois, E. N ishida, G. Almouzni, O. Bensaude. 1997. Phosphorylation of the RN A polymerase II largest subunit during Xenopus laevis oocyte maturation. Mol. Cell. Biol. 17: 1434-1440.

Buck, V., P. Russel, and J.B.A. Millar. 1995. Identification of a cdk-activating kinase in fission yeast. EMBO J. 14: 61736183.

Cisek, L.J. and J.L. Corden. 1989. Phosphorylation of RN A polymerase by the murine homologue of the cell-cycle control protein cdc2. Nature 339: 679-684.

Cismowski, M.J., G.M. Laff, M.J. Solomon, and S. Reed. 1995. KIN 28 Encodes a C-terminal domain kinase that controls mRN A transcription in Saccharomyces cerevisiae but lacks cyclin-dependent kinase-activating kinase (CAK) activity. Mol. Cell. Biol. 15: 2983-2992.

Coleman, T.R. and W.G. Dunphy. 1994. Cdc2 regulatory factors. Curr. Opin. Cell Biol. 6: 877-882.

Conaway, J.W., D. Reines, and R.C. Conaway. 1990. Transcription initiated by RNA polymerase II and purified transcription factors from liver. Cooperative action of transcription factors tau and epsilon in initial complex formation. J. Biol. Chem. 265: 7552-7558.

Dahmus, M.E. 1996. Reversible phosphorylation of the C-terminal domain of RNA polymerase II. J. Biol. Chem. 271: 19009-19012.

Devault, A., A.-M . M artinez, D. Fesquet, J.-C. Labbe, N. M orin, J.-P. Tassan, E.A. N igg, J.-C. Cavadore, and M. Doree. 1995. MAT1 a new RING finger protein subunit stabilizing cyclin $\mathrm{H}$-cdk7 complexes in starfish and Xenopus CAK. EMBO J. 14: 5027-5036.

Drapkin, R. and D. Reinberg. 1994 The multifunctional TFIIH complex and transcriptional control. Trends Biochem. Sci. 19: 504-508.

Drapkin, R., J.T. Reardon, A. Ansari, J.-C. Huang, L. Zawel, K. A hn, A. Sancar, and D. Reinberg. 1994. Dual role of TFIIH in DNA excision repair and in transcription by RNA polymerase II. Nature 368: 769-772.

Drapkin, R., G. Le Roy, H. Cho, S. Akoulitchev, and D. Reinberg. 1996. Human cyclin-dependent kinase-activating kinase exists in three distinct complexes. Proc. Natl. Acad. Sci. 93: 6488-6493.

Elledge, S.J. and J.W. Harper. 1994. Cdk inhibitors: On the threshold of checkpoints and development. Curr. O pin. Cell Biol. 6: 847-852.

Fink, K. and G. Turnock. 1977. Synthesis of transfer RN A during the synchronous nuclear division cycle in Physarum polycephalum. Eur. J. Biochem. 80: 93-96.

Fisher, R.P. and D.O. Morgan. 1994. A novel cyclin associates with $\mathrm{MO15/CDK7}$ to form the CDK-activating kinase. Cell 
78: $713-724$.

Fisher, R.P., P. Jin, H.M. Chamberlin, and D.O. Morgan. 1995. Alternative mechanisms of CAK assembly require an assembly factor or an activating kinase. Cell 83: 47-57.

Gautier, J., J. M inshull, M. Lohka, M. Glotzer, T. Hunt, and J.L. Maller. 1990. Cyclin is a component of maturation-promoting factor from Xenopus. Cell 60: 487-494.

Goodrich, J.A. and R. Tjian. 1994. Transcription factors IIE and $\mathrm{IIH}$ and ATP hydrolysis direct promoter clearance by RNA polymerase II. Cell 77: 145-156.

Gottesfeld, J.M. and D.J. Forbes. 1997. Mitotic repression of the transcriptional machinery. Trends Biochem. Sci. 22: 197202.

Gottesfeld, J.M., V.J. Wolf, T. Dang, D.J. Forbes, and P. Hartl. 1994. Mitotic repression of RNA polymerase III transcription in vitro mediated by phosphorylation of a TFIIIB component. Science 263: 81-84.

Johnson, T.C. and J.J. Holland. 1965. Ribonucleic acid and protein synthesis in mitotic HeLa cells. J. Cell Biol. 27: 565574.

Johnston, L.H., J.H. White, A.L. Johnson, G. Lucchini, and P. Plevani. 1987. The yeast DNA polymerase I transcript is regulated in both the mitotic cell cycle and in meiosis and is also induced after DNA damage. Nucleic Acids Res. 15: 5017-5030.

Kinoshita, N., H. Ohkura, and M. Yanagida. 1990. Distinct, essential roles of type 1 and $2 \mathrm{~A}$ protein phosphatases in the control of the fission yeast cell division cycle. Cell 63: 405415.

King, R.W., P.K. Jackson, and M.W. Kirschner. 1994. Mitosis in transition. Cell 79: 563-571.

Kumar, K.P., S. Akoulitchev, and D. Reinberg. 1998. Promoterproximal stalling results from the inability to recruit transcription factor $\mathrm{IIH}$ to the transcription complex and is a regulated event. Proc. Natl. Acad. Sci. 95: 9767-9772.

Labbe, J.C., J.P. Capony, D. Caput, J.C. Cavadore, J. Derancourt, M. Kaghad, J.M. Lelias, A. Picard, and M. Doree. 1989. M PF from starfish oocytes at first meiotic metaphase is a heterodimer containing one molecule of cdc2 and one molecule of cyclin B. EMBO J. 8: 3053-3058.

Labbe, J.C., J.C. Cavadore, and M. Doree. 1991. M phase-specific cdc2 kinase: preparation from starfish oocites and properties. Methods Enzymol. 200: 291-301.

Labbe, J.-C., A.-M. Martinez, D. Fesquet, J.-P. Capony, J.-M. Darbon, J. Derancourt, A. Devault, N. M orin, J.-C. Cavadore, and M. Doree. 1994. p40M 015 associates with a p36 subunit and requires both nuclear translocation and Thr 176 phosphorylation to generate cdk-activating kinase activity in Xenopus oocytes. EMBO J. 13: 5155-5164.

Larochelle, S., J. Pandur, R.P. Fisher, H.K. Salz, and B. Suter. 1998. Cdk7 is essential for mitosis and for in vivo Cdk-activating kinase activity. Genes \& Dev. 12: 370-381.

LeRoy, G., R. Drapkin, L. Weis, and D. Reinberg. 1998. Immunoaffinity purification of the human multisubunit transcription factor IIH. J. Biol. Chem. 273: 7134-7140.

Long, J.J., A. Leresche, R.W. Kriwaski, and J.M. Gottesfeld. 1998. Repression of TFIIH Transcriptional activity and TFIIH-associated cdk7 kinase activity at mitosis. Mol .Cell. Biol. 18: 1467-1476.

Lu, H., O. Flores, R. Weinmann, and D. Reinberg. 1991. The nonphosphorylated form of RNA polymerase II preferentially associates with the preinitiation complex. Proc. Natl. Acad. Sci. 88: 10004-10008.

Lu, H., L. Zawel, L. Fisher, J.-M. Egly, and D. Reinberg. 1992. Human general transcription factor IIH phosphorylates the C-terminal domain of RN A polymerase II. Nature 358: 641-
645.

Luo, K. and H.F. Lodish. 1997. Positive and negative regulation of type II TGF-beta receptor signal transduction by autophosphorylation on multiple serine residues. EMBO J. 16: 19701981.

Ma, D., I. Olave, A. M erino, and D. Reinberg. 1996. Separation of the transcriptional coactivator and antirepression functions of transcription factor IIA. Proc. Natl. Acad. Sci. 93: 6583-6588.

Makela, T., J.D. Parvin, J. Kim, L.J. Huber, P.A. Sharp, and R.A. Weinberg. 1995. A kinase-deficient transcription factor TFIIH is functional in basal and activated transcription. Proc. Natl. Acad. Sci. 92: 5174-5178.

Manley, J., A. Fire, A. Cano, P. Sharp, and M. Gefter. 1980. DNA-dependent transcription of adenovirus genes in a soluble whole-cell extract. Proc. Natl. Acad. Sci. 77: 38553859.

M arinoni, J.-C., W. Vermeulen, P. M iniou, Y. Lutz, G. Weeda, T. Seroz, D.M. Gomez, J.H.J. Hoejimakers, and J.M. Egly. 1997. Cloning and characterization of p52, the fifth subunit of the core of the transcription/DN A repair factor TFIIH. EMBO J. 16: 1093-1102.

Martinez, A.-M., M. Afshar, F. Martin, J.-C. Cavadore, J.-C. Labbe, and M. Doree. 1997. Dual phosphorylation of the Tloop in cdk7: Its role in controlling cyclin $\mathrm{H}$ binding and CAK activity. EMBO J. 16: 343-354.

Nigg, E.A. 1993. Targets of cyclin-dependent protein kinases. Curr. Opin. Cell. Biol. 5: 187-193.

N orbury, C. and P. Nurse. 1992. Animal cell cycles and their control. Annu. Rev. Biochem. 61: 441-470.

O'Brien, T. and J.T. Lis. 1991. RN A polymerase II pauses at the $5^{\prime}$ end of the transcriptionally induced Drosophila hsp70 gene. Mol. Cell. Biol. 11: 5285-5290.

Orphanides, G., T. Lagrange, and D. Reinberg. 1996. The general transcription factors of RNA polymerase II. Genes \& Dev. 10: 2657-2683.

Parsons, G.G. and C.A. Spencer. 1997. Mitotic repression of RN A polymerase II transcription is accompanied by release of transcription elongation complexes. Mol. Cell. Biol. 17: 5791-5802.

Reardon, J.T., H. Ge, E. Gibbs, A. Sancar, J. Hurwitz, and Z.Q. Pan. 1996. Isolation and characterization of two human transcription factor IIH (TFIIH)-related complexes: ERCC2/CAK and TFIIH. Proc. Natl. Acad. Sci. 93: 6482-6487.

Reed, S.I. 1992. The role of p34 kinases in the $\mathrm{G} 1$ to S-phase transition. Annu. Rev. Cell Biol. 8: 529-561.

Roeder, R.G. 1991. The role of general transcription factors in transcription by RNA polymerase II. Trends Biochem. Sci. 16: 402-408.

Rossignol, M., I. Kolb-Cheynel, and J.M. Egly. 1997. Substrate specificity of the cdk-activating kinase (CAK) is al tered upon association with TFIIH. EMBO J. 16: 1628-1637.

Russo, A., P.D. Jeffrey, and N.P. Pavletich. 1996. Structural basis of cyclin-dependent kinase activation by phosphorylation. Nat. Struct. Biol. 3: 696-700.

Sancar, A. 1996. DNA excision repair. Annu. Rev. Biochem. 65: 43-81.

Segil, N., M. Guermah, A. Hoffmann, R.G. Roeder, and N. Heintz. 1996. Mitotic regulation of TFIID inhibition of activator-dependent transcription and changes in subcellular localization. Genes \& Dev. 10: 2389-2400.

Shaeffer, L., R. Roy, S. Humbert, V. M oncollin, W. Vermeulen, J.H. Hoeijmakers, P. Chambon, and J.M. Egly. 1993. DNA repair helicase: A component of BTF2 (TFIIH) basic transcription factor. Science 260: 58-63.

Shermoen, A.W. and P.H. O'Farrell. 1991. Progression of the cell 


\section{Akoulitchev and Reinberg}

cycle through mitosis leads to abortion of nascent transcripts. Cell 67: 303-310.

Shuttleworth, J., R. Godfrey, and A. Colman. 1990. p40M 015, a cdc2-related protein kinase involved in negative regulation of meiotic maturation of Xenopus oocytes. EMBO J. 9: 32333240.

Svejstrup, J.Q., Z. Wang, W.J. Feaver, X. Xu, D.A. Bushnell, T.F. Donahue, E.C. Friedberg, and R.D. Kornberg. 1995. Different forms of TFIIH for transcription and DNA Repair: HoloTFIIH and a nucleotide excision repairosome. Cell 80: 2128.

Svejstrup, J.Q., P. Vichi, and J.M. Egly.1996 The multiple roles of transcription/repair factor TFIIH. Trends Biochem. Sci. 21: 346-350.

Tassan, J.-P., S. Schultz, J. Bartek, and E.A. Nigg. 1994. Cell cycle analysis of the activity, subcellular localization, and subunit composition of human CAK (CDK-activating kinase). J. Cell Biol. 127: 467-478.

Valay, J.-G., M. Simon, M.-F. Dubois, O. Bensaude, C. Facca, and G. Faye. 1995. The KIN 28 gene is required both for RN A polymerase II mediated transcription and phosphorylation of the Rpb1p CTD. J. Mol. Biol. 249: 535-544.

Weeks, J.R., S.E. Hardin, J. Shen, L.M. Lee, and A.L. Greenleaf. 1993. Locus-specific variation in phosphorylation state of RN A polymerase II in vivo: Correlations with gene activity and transcript processing. Genes \& Dev. 7: 2329-2344.

Yankulov, K.Y. and D.L. Bentley. Regulation of CDK7 substrate specificity by MAT1 and TFIIH. 1997. EMBO J. 16: 16381646.

Zawel, L., H. Lu, L.J. Cisek, J.L. Corden, and D. Reinberg. 1993. The cycling of RN A Polymerasell during transcription. Cold Spring Harbor Symp. Quant. Biol. 58: 187-198.

Zhou, Q., P.M. Lieberman, T.G. Boyer, and A.J. Berk. 1992. Holo-TFIID supports transcriptional stimulation by diverse activators and from a TATA-less promoter. Genes \& Dev. 6: 1964-1974.

Zhu, Y., T. Pe'ery, J. Peng, Y. Ramanathan, N. Marshall, T. Marshall, B. Amendt, M.B. Mathews, and D. Price. 1997. Transcription el ongation factor $\mathrm{P}-\mathrm{TEFb}$ is required for HIV-1 Tat transactivation in vitro. Genes \& Dev. 11: 2622-2632 


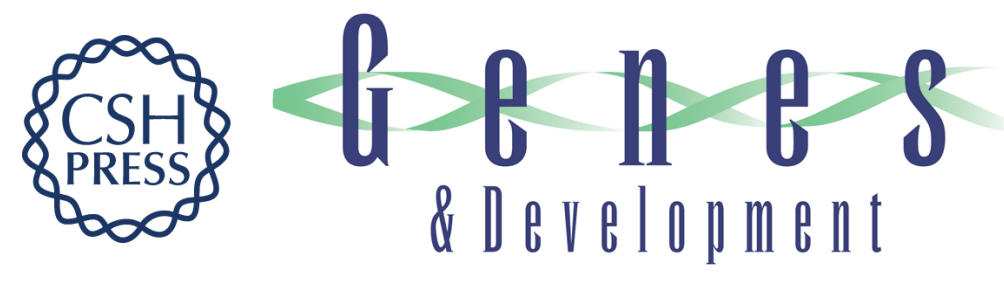

\section{The molecular mechanism of mitotic inhibition of TFIIH is mediated by phosphorylation of CDK7}

Sasha Akoulitchev and Danny Reinberg

Genes Dev. 1998, 12:

Access the most recent version at doi:10.1101/gad.12.22.3541

References This article cites 64 articles, 31 of which can be accessed free at: http://genesdev.cshlp.org/content/12/22/3541.full.html\#ref-list-1

License

Email Alerting Receive free email alerts when new articles cite this article - sign up in the box at the top Service right corner of the article or click here.

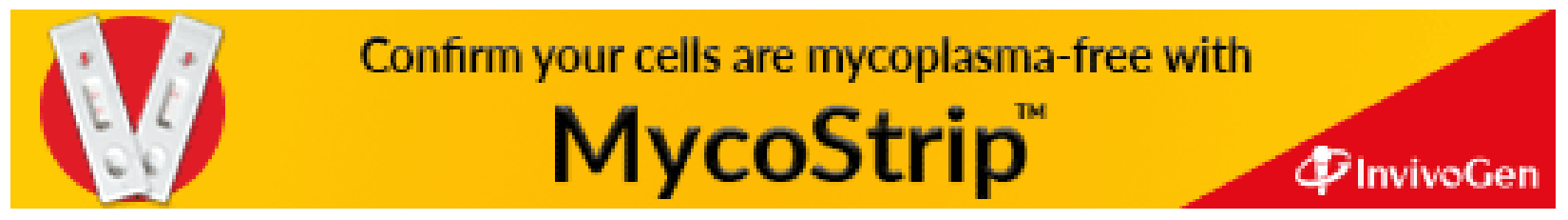

